\title{
Erratum
}

\section{Erratum to: On the pairs of multiplicative functions satisfying some relations}

NADER L. BASSILY AND IMRE KÁtAI

\section{Erratum to: Aequat. Math. 55 (1998), 1-14 DOI 10.1007/PL00000074}

In the original article we asserted (Theorem 1):

Theorem 1. If $f$ and $g$ are complex valued multiplicative functions and

$$
g(2 n+1)-A f(n) \rightarrow 0(n \rightarrow \infty)
$$

with some constant $A \neq 0$, then either $f(n) \rightarrow 0(n \rightarrow \infty)$, or $A=$ $f(2), f(n)=n^{s}$ and $f(n)=g(n)$ for odd integers $n$.

This assertion is incorrect, as the following example shows:

Let $F(n)=(-1)^{n-1}(n=1,2, \ldots), G(2 n+1)=\chi_{4}(2 n+1)$, where $\chi_{4}$ is the nonprincipal Dirichlet character $(\bmod 4)$. Then $F(n)$ and $G(n)$ are multiplicative functions $(G(n)$ can be arbitrary on the powers of 2$)$, furthermore

$$
G(2 n+1)=-F(n)(n \in \mathbb{N}) .
$$

The failure comes from an incorrect assertion made in the proof of Lemma 8.

Let $H(n)=\frac{f(n)}{g(n)}$. Following the argument of the proof of Lemma 8 , we obtain that $H(3)=H(Q)$ if $Q \equiv 3,11(\bmod 12)$, thus $H(3)=$ $H(3(4 n+1))=H(3) H(4 n+1)$ if $(3,4 n+1)=1$. For $Q=11$ we have $H(3)=H(11)$. Since $\nu, \mu \equiv 3(\bmod 4),(\nu, \mu)=1$ implies that $H(\nu \mu)=$ $H(\nu) H(\mu)=1$, if $(\nu \mu, 3)=1$, it can occur only if $H(n)=$ constant if $n$ runs

The online version of the original article can be found under doi:10.1007/PL00000074. 
over the integers $n \equiv 3(\bmod 4),(n, 3)=1$. Since $3^{1+2 t} \equiv 3(\bmod 12)$, choosing $Q=3^{1+2 t}, H(3)=H\left(3^{1+2 t}\right)$. Let $Q=11 \cdot 3^{2 t}$. Then $Q \equiv 3(\bmod 12)$, and so $H(3)=H(11) H\left(3^{2 t}\right)$, consequently $H\left(3^{2 t}\right)=1(t=1,2, \ldots)$.

Let $H(n)=\xi$ if $n \equiv 3(\bmod 4)$. There are two possibilities:

(A): $\xi=1$

(B): $\xi=-1$.

In case (A) follow the further argumentation of Lemma 8, consequently the theorem formulated in [1] is true.

In Case (B) define

$$
g_{1}(n)=\frac{g(n)}{G(n)} \text { for odd } n, \quad f_{1}(n)=\frac{f(n)}{F(n)} .
$$

From (1.1) we obtain that

$$
g_{1}(2 n+1)+A f_{1}(n) \rightarrow 0(n \rightarrow \infty)
$$

and for $H_{1}(n)=\frac{g_{1}(n)}{f_{1}(n)}$ we obtain that $H_{1}(n)=1$ for every odd $n$.

Consequently the following assertion is true.

Theorem. Let $f, g$ be complex valued multiplicative functions satisfying (1.1) with some constant $A \neq 0$. Then the following possibilities can occur:

(1) $\quad f(n) \rightarrow 0(n \rightarrow \infty)$ and $g(m) \rightarrow 0(m \rightarrow \infty,(m, 2)=1)$

(2) $\quad f(n)=n^{s}, g(m)=m^{s}(n, m \in \mathbb{N},(m, 2)=1, A=f(2)$

(3) $\frac{f(n)}{g(n)}=\chi_{4}(n)$ for odd $n$.

Let $g_{1}(2 n+1)=\frac{g(2 n+1)}{G(2 n+1)}, f_{1}(n)=\frac{f(n)}{F(n)}$. Then $f_{1}(n)=n^{s}, g_{1}(m)=m^{s}(n$, $m \in \mathbb{N},(m, 2)=1)$.

The authors express their sincere thanks to Dr. Bui Minh Phong who called their attention to the failure.

\author{
N. L. Bassily \\ 6 EL Mamalik Str. 8 \\ Roxy, Heliopolis \\ Egypt \\ I. Kátai \\ Eötvös Loránd University \\ Computer Algebra Dept. \\ Múzeum krt. 6-8 \\ H-1088 Budapest \\ Hungary
}

